\title{
Enhanced surface capacitance of cylindrical micropillar arrays
}

\author{
Assaf Ya'akobovitz \\ Mechanosynthesis Group, Department of Mechanical Engineering, University of \\ Michigan, Ann Arbor, MI 48109, USA \\ A. John Hart \\ Mechanosynthesis Group, Department of Mechanical Engineering, University of \\ Michigan, Ann Arbor, MI 48109, USA \\ Mechanosynthesis Group, Department of Mechanical Engineering and Laboratory for \\ Manufacturing and Productivity, Massachusetts Institute of Technology, Cambridge, MA \\ 02139, USA
}

\begin{abstract}
While surface capacitance is utilized widely for sensing and actuation in miniaturized systems, relatively little attention has been paid to its enhancement using engineered surface topography. We present how an engineered surface topography, specifically a cylindrical micropillar array, gives a significant enhancement of the surface capacitance compared to a flat surface of the same area. Arrays of silicon micropillars were fabricated and measured, and the results were validated using both an approximate analytical model and a numerical finite element model. At large gaps (hundreds of microns) between a flat probe and the top surface of the array, the capacitance is comparable to that of a flat surface, while at smaller gaps $(<200 \mu \mathrm{m})$ the contribution of the side surface leads to enhancement of the capacitance. We demonstrate that parametric variation of the array geometry and gap can result in greater than $100 \%$ enhancement, while using materials and dimensions compatible with standard microfabrication processes. Therefore, we expect that this engineered surface design can improve the performance of traditional capacitance probes as well as emerging applications such as flexible tactile sensors.
\end{abstract}

Keywords: Capacitance, electrostatic, sensing, microfabrication, MEMS 


\section{Introduction}

Due to their performance and compatibility with standard microfabrication processes, micro-scale electrostatic capacitors have been the focus of widespread research and industrial development. Capacitive comb-finger arrays are a building block of mass-market MEMS devices including accelerometers [1], while studies in scanning probe microscopy have pushed the ultimate limits by which capacitance can be used to measure displacement and force $[2,3]$. Along with continued advances in microfabrication processing which affords integration of capacitive sensors with small gaps, recent applications include tactile [4] and proximity [5] sensors as well as advanced energy harvesting devices [6], and robotic systems [7].

In these applications the surface topography of the electrodes influences the capacitance. In the case of capacitive devices for energy storage, larger area increases the amount of charge stored; in the case of displacement sensors, a higher capacitance enables resolution of smaller incremental motions. Nevertheless, most known micro-scale capacitive devices as well as centimeter-scale capacitance probes use parallel plate geometries [5, 7], and fringing fields, originated from the edges, are often neglected. The influence of the fringe capacitive field, which can increase the capacitance, was investigated for several geometries common in MEMS such as micro beams [8], comb finger actuators [9] and perforated parallel plates [10]. The effect of surface roughness on capacitance and electrostatic forces was studied, and it was found that rough surfaces contribute to the electrostatic force for large gaps and reduce it for smaller gaps [11]. Moreover, for rough surfaces the relation between the capacitance and wear [12] and pull-in behavior [11] was investigated.

Nevertheless, to our knowledge there has not been a study of how to modify the topography of a surface using microfabrication, to give a significant enhancement of capacitance. In an effort to demonstrate this principle, we present how an exemplary microfabricated topography, specifically an array of cylindrical silicon micropillars, can provide significant enhancement to surface capacitance using a standard fabrication process and parallel plate measurement configuration.

\section{Theory and modeling}

We consider a cylindrical micropillar array on a square grid, as shown schematically in Fig. 1. Capacitance is derived from the solution of the 
well-known electrostatic boundary value problem (ES-BVP), expressed as follows

$$
\begin{aligned}
& \Delta \varphi=0 \text { in } \Omega, \\
& \varphi=G \text { on } \Gamma_{G} \\
& -\frac{\partial \varphi}{\partial n}=H \text { on } \Gamma_{H} \\
& \lim _{x, y, z \rightarrow \infty} \varphi=0
\end{aligned}
$$

where $\varphi$ is the potential in the air domain $\Omega, \Delta$ is Laplace operator, $G$ is a known potential on the boundary $\Gamma_{G}, H$ is a known electric field perpendicular to the boundary $\Gamma_{H}$, and $n$ is a coordinate normal to the surface. The capacitance of a capacitor of area $A$ is derived from the solution of Eq. (1) and can be calculated as

$$
C=\frac{Q}{V}=\frac{\epsilon_{0} \epsilon_{r}}{V} \int_{A} \frac{\partial \varphi}{\partial n} d A
$$

Here $Q$ is the electric charge and $\epsilon_{0}$ and $\epsilon_{r}$ are the dialectic permittivities of vacuum and air, respectively. Alternatively, the capacitance can be expressed as

$$
C=\frac{\epsilon_{0} \epsilon_{r} A}{g}
$$

where $g$ is the length of the field lines.

\subsection{Numerical model}

First, the ES-BVP shown in Eq. (1) was solved numerically [10] using finite element (FE) simulation (ADINA software). Taking advantage of the symmetry of the square lattice of micropillars, only a model of one fourth of a pillar was required, as shown in Fig. 2. The potential distribution in the air and the charge distribution on a pillar having potential difference of $1 \mathrm{~V}$, dimensions of Array 1 (Table 1 ) and $g=50 \mu \mathrm{m}$ is shown in Fig. 2(a); the capacitance was calculated using Eq. (2). Figure 2(b) demonstrates that the majority of charge is distributed around the top edge of the pillar, both on the top and sidewall surfaces, emphasizing the significant contribution of the fringing fields to the capacitance. Capacitance values derived from this model are shown as green stars in Fig. 2(c) as a function of the gap.

\subsection{Analytical model}

For further understanding of the array dimensions and for parametric study purposes, an approximate solution of the capacitance was derived analytically, considering the superposition of contributions from the pillar top, 


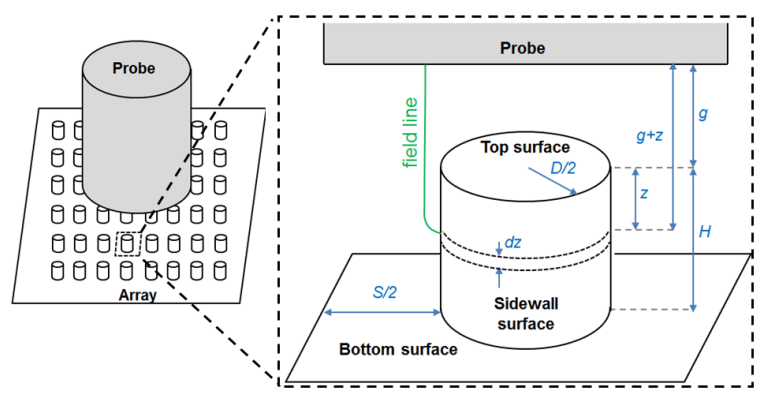

Figure 1: Schematic of the micropillar array, with a close-up diagram of a unit cell of the array. A ring-shape element of area $d A$ is shown by dashed lines, the vertical gap between the pillar and the probe is $g$, the pillar height is $H$, and the spacing is $S$.

Table 1: Dimensions of the silicon micropillar arrays used for capacitance measurements.

\begin{tabular}{|l|l|l|l|}
\hline Array number & $\begin{array}{l}\text { Diameter } \\
D[\mu m]\end{array}$ & $\begin{array}{l}\text { Spacing } \\
S[\mu m]\end{array}$ & $\begin{array}{l}\text { Height } \\
H[\mu m]\end{array}$ \\
\hline 1 & 94 & 170 & 51 \\
\hline 2 & 392 & 67 & 51 \\
\hline 3 & 170 & 185 & 51 \\
\hline 4 & 84 & 34 & 51 \\
\hline
\end{tabular}

sidewalls, and bottom surfaces. We approximated the length of the field lines originating from the sidewalls as the vertical distance to the probe $(g+z)$, as shown in Fig. 1. Note that neglecting the length of the curved part of the field line is not valid for small gaps (a few microns or less). Also note that this field line length assumption is valid in cases where the spacing between the pillars is sufficiently large (around $100 \mu \mathrm{m}$ or more, as discussed below), so that the field lines from the sidewalls of adjacent pillars do not interact with one others. 
(a)
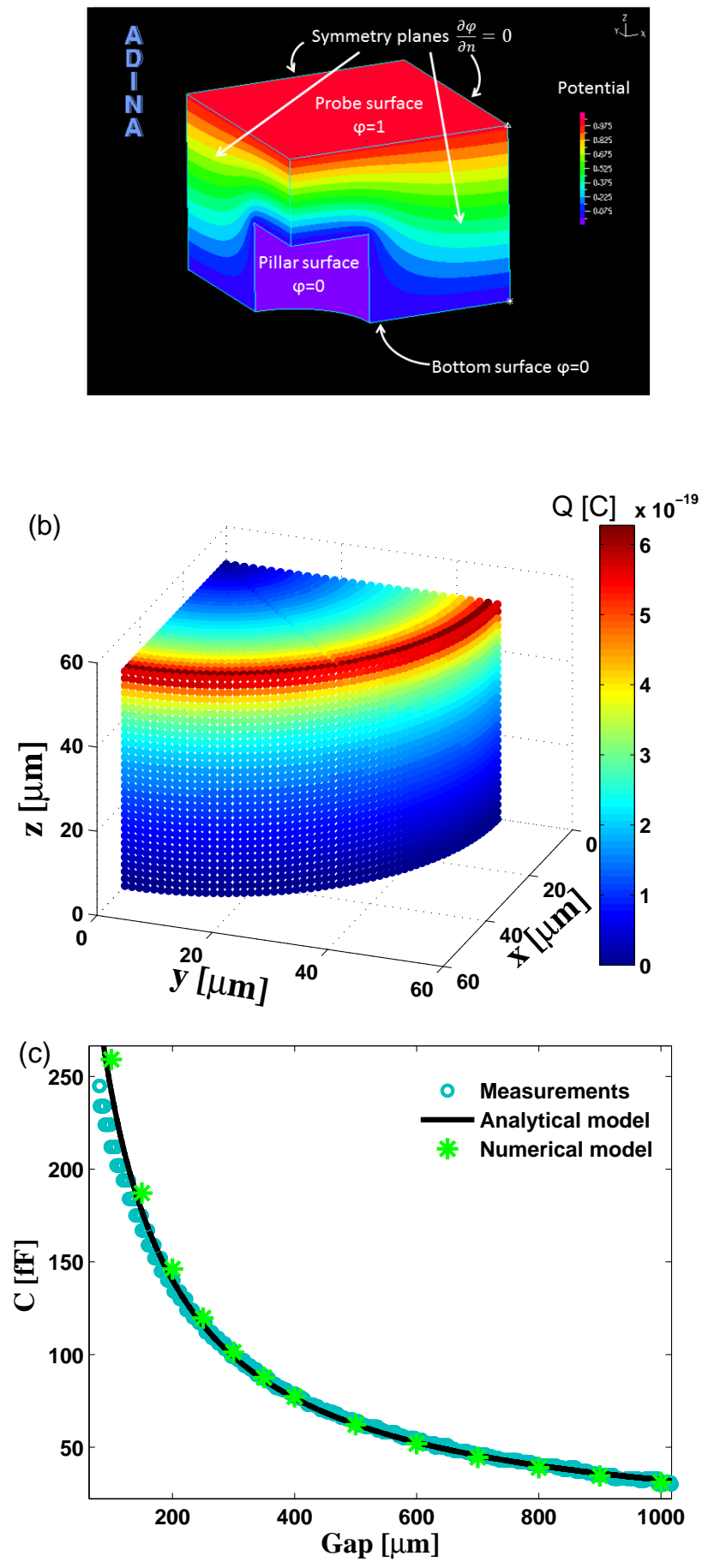

Figure 2: Results for Array 1 (Table 1) at $g=50 \mu \mathrm{m}$. Potential difference between the probe and the pillar was taken as 1V. (a) Potential distribution of the air between a pillar and the probe obtained from the numerical model. (b) Charge distribution on the surface of the pillar obtained from the numerical model. (c) Measured and calculated capacitance versus gap. The gap is measured between the probe bottom surface and the top surface of the micropillar array. 
Using Eq. (3), we derived the capacitance of a ring shaped element of area $d A=\pi D d z$ (Fig. 1) as

$$
d C_{\text {side }}=\frac{\epsilon_{0} \epsilon_{r} d A}{g+z}
$$

The total capacitance of the sidewall is therefore

$$
C_{\text {side }}=\int_{z=g}^{g+H} d C_{\text {side }}=\epsilon_{0} \epsilon_{r} \pi D \ln \left(\frac{g+H}{g}\right)
$$

We then approximate the horizontal (top and bottom) surfaces as parallel plate capacitors such that

$$
\begin{gathered}
C_{\text {top }}=\frac{\epsilon_{0} \epsilon_{r} \pi D^{2}}{4 g} \\
C_{\text {bottom }}=\frac{\epsilon_{0} \epsilon_{r}\left((D+S)^{2}-\left(\pi D^{2} / 4\right)\right)}{g+H}
\end{gathered}
$$

where $S$ is the spacing between the closest points on adjacent pillars, Fig. 1. The horizontal surfaces do not behave as a classical parallel plate (see charge distribution in Fig. 2), however, this gives a good approximate estimation to their capacitance contribution (errors with respect to the numerical model and experimental measurements are discussed below).

The total capacitance of an array is therefore

$$
C=N\left(C_{\text {side }}+C_{\text {top }}+C_{\text {bottom }}\right),
$$

where $N$ is the numbers of pillars underneath the probe.

The micropillar array capacitance estimated using the analytical model is shown for array 1 by the solid black line in Fig. 2(c). Despite our assumptions, here this model presents excellent agreement (low RMS error, see below) with the numerical and experimental results. Note that for small gaps and spacing, the error increases for the reasons stated above.

\section{Experiment}

\subsection{Fabrication}

Several micropillar arrays were fabricated and characterized. The fabrication process is described schematically in Fig. 3. A 
(a)

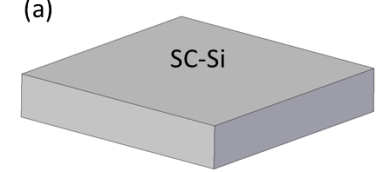

(b)
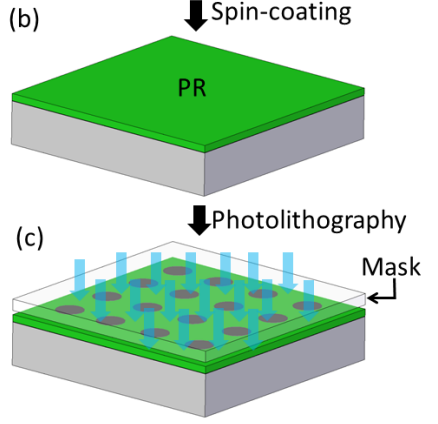

(d)

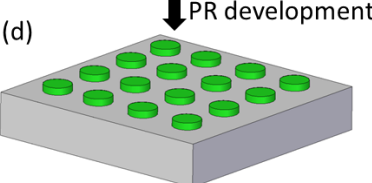

(e) $\quad$ DRIE

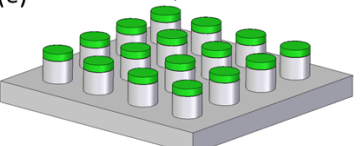

Acetone,

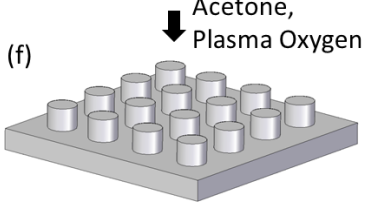

Figure 3: Fabrication process. (a) SC-Si wafer was used as a starting material. (b) Spincoating of positive PR. (c) Exposure of the PR using a hard contact mask. UV light is shown as light blue arrows. (d) Development of the PR. (e) DRIE. (f) Removal of the PR by means of acetone washing and oxygen plasma.

heavily doped p-type single crystal silicon (SC-Si) wafer was used as a starting material, as shown in Fig. 3(a). This wafer has good electrical conductivity that is necessary for the capacitance measurements. First, the wafer was spin-coated with a positive photoresist (PR), as described in Fig. 3(b). Then, we have fabricated a mask with arrays of circles and used it (hard contact exposure) to selectively expose the PR to UV light (Fig. 3(c)), followed by PR development, shown in Fig. 3(d). Next, in order to get high aspect ratio micro-pillars arrays with straight sidewalls, deep reactive ion etching (DRIE) of the wafer was carried out, as illustrated in Fig. 3(e). Finally, the PR was removed first by washing the wafer with acetone and then by oxygen plasma, Fig. 3(f). A scanning electron microscope image of a typical fabricated array is shown in Fig. 4. The dimensions of the micropillars characterized in this work are listed in Table 1, and include arrays with different values of diameters and spacings. 


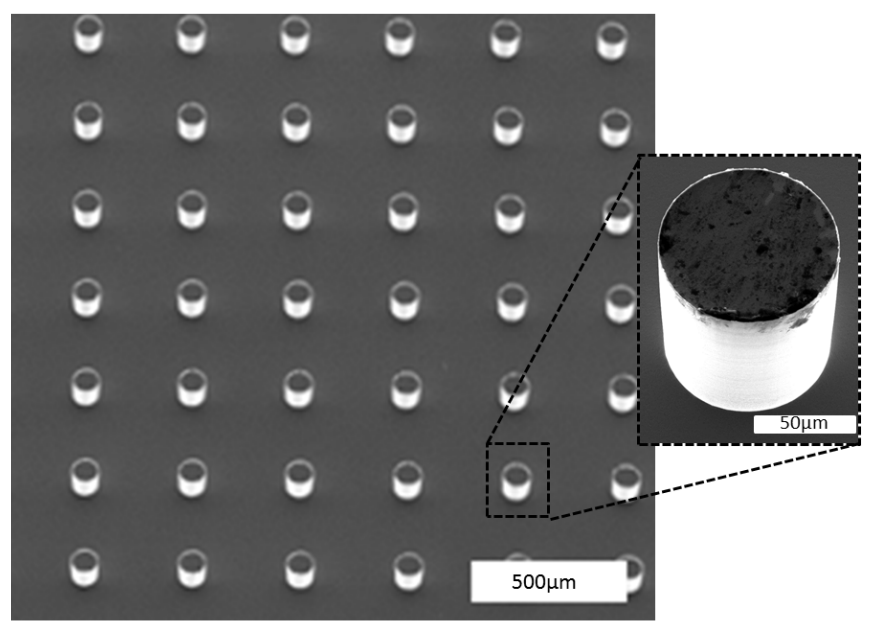

Figure 4: An SEM image of one of the fabricated micro-pillars arrays. A single pillar is shown in the inset.

\subsection{Experimental setup}

The system used for characterization is shown schematically in Fig. 5. The system includes a capacitance probe (Capacitec HPB-75 Button Probe) attached to a custom-built linear voicecoil actuator. The probe position is controlled by a computer using LabView interface, which was also used to store the capacitance data (HP4280A). Experiments were carried out on a floating table (Thorlabs SDA7590), so that the influence of environmental vibrations was negligible. The experiments were imaged optically using a high resolution camera (Edmund Optics NT85-871, pixel size is $2 \mu \mathbf{m} \times 2 \mu \mathbf{m}$,$) . Side view optical images were used to determine the$ initial gap and then the probe displacement was measured using a linear optical encoder (Renishaw model REF1000E25A, step is size is $20 \mathrm{~nm}$ ) attached to the actuator. The gap $(g)$ is measured from the plane containing the top surfaces of the micropillars and the error in gap measurement was determined to be less than one micron. 


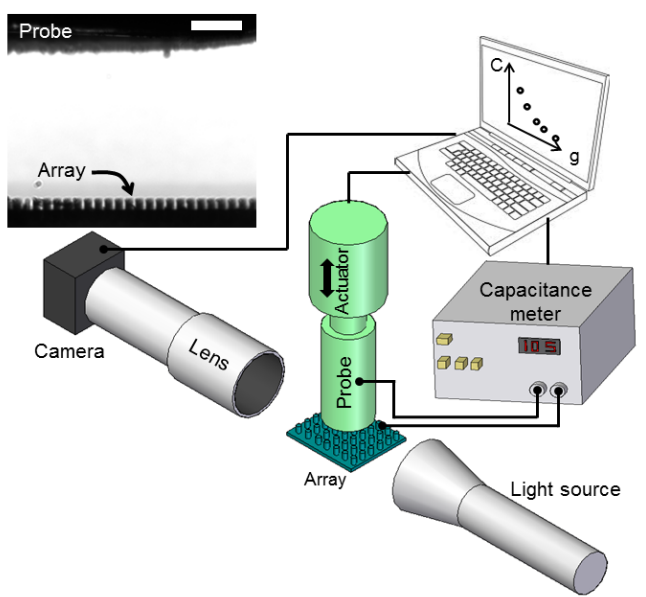

Figure 5: Schematic of Measurement setup of capacitance between the flat probe and the silicon micropillar array. A vertically movable flat probe is connected to a vertical precision actuator and a capacitance meter. Inset: Optical image of the probe and array (side view). Bar is $500 \mu \mathrm{m}$. 


\subsection{Results}

The measured capacitance of Array 1 versus the gap is shown as light blue circles in Fig. 2(c) and presented excellent agreement with the predicted capacitance, as the numerical and analytical models demonstrated an RMS error relative to the experimental measurements on the order of $0.1 \mathrm{fF}$ and $1 \mathrm{fF}$, respectively. As a reference, the capacitance of a flat $\mathrm{Si}$ surface was measured, and the capacitance enhancement $\left(C / C_{\text {flat }}\right)$ for all four arrays is shown in Fig. 6. Note that the measured capacitance of the flat surface is slightly lower than that of a calculated flat surface.

In cases where the micropillar topography results in capacitance enhancement (Arrays 1,2 and 3) the relative enhancement is inversely proportional to the gap, while as the gap increases the capacitance of the arrays approaches that of a flat surface $\left(C / C_{\text {flat }} \rightarrow 1\right)$. The relative contributions of the surface components derived from the analytical model are presented for Array 1 in the inset of Fig. 6. At small gaps $(\sim 10 \mu \mathrm{m})$ the effect of the sidewall surfaces is significant and as a result the capacitance enhancement is higher. On the other hand, at larger gaps, the bottom surface is dominant and therefore the capacitance is closer to that of a flat surface. Notice that for certain geometries the micropillar arrays results in attenuation (enhancement $<1$ ) of the capacitance with respect to that of a flat surface, as demonstrated by Array 4 , where the spacing between the pillars is too small, see discussion below.

\section{Parametric study of array dimensions}

The analytical model was now used to show how the capacitance scales with the micropillar array geometry, revealing how to design arrays to achieve significant enhancement of the surface capacitance. First, the validity of the analytical model was evaluated. The capacitances of several arrays were calculated using both the numerical and analytical models and the relative errors were extracted, Fig. 7. We find that for diameters and spacing larger than $100 \mu \mathrm{m}$ the error of the analytical model is less than $10 \%$. In this range of diameters and spacings the interactions between the adjacent micropillars are negligible and our assumptions regarding field line length and distribution are valid. In cases where the pillars are close to one another, they interfere with each other, which results in attenuation of the capacitance, as demonstrate by Array 4 in Fig. 6.

The capacitance enhancement is derived by dividing Eq. (8) by the capacitance of a flat surface 


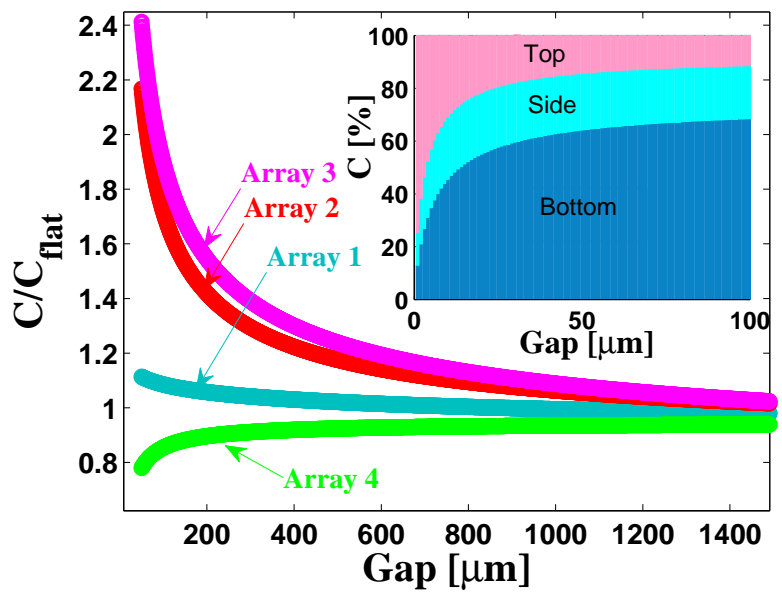

Figure 6: Measured capacitance enhancement for all four arrays (Table 1), relative to a flat surface of the same area at the same gap. Inset: calculated contributions of the top, side, and bottom surfaces, versus gap, for Array 1.
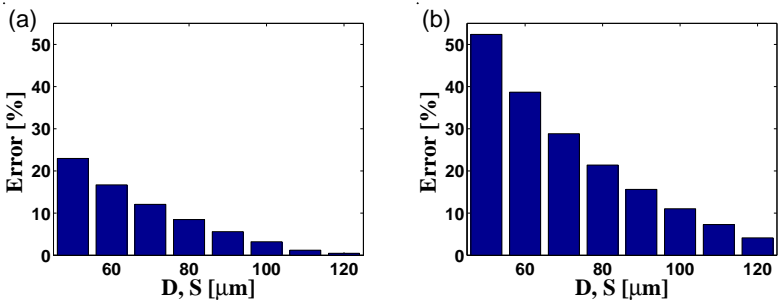

Figure 7: Calculated relative error of the approximated analytical model with respect to the numerical model. The diameter and spacing were chosen to be equal $D=S$, the height was $H=51 \mu \mathrm{m}$ and the gap was (a) $g=20 \mu \mathrm{m}$ and (b) $g=100 \mu \mathrm{m}$. 


$$
C_{\text {flat }}=\frac{\epsilon_{0} \epsilon_{r}(S+D)^{2}}{g}
$$

such that the capacitance enhancement is

$$
\frac{C}{C_{\text {flat }}}=\frac{1}{(1+S / D)^{2}}\left(\frac{(1+S / D)^{2}-\pi / 4}{(1+H / g)}+(g / D) \pi \ln (H / g+1)+\pi / 4\right)
$$

The enhancement is determined by the dimensionless parameters $S / D, H / g$ and $g / D$, and for given values of $g$ and $H$, there always exists a combination of diameter and spacing that results in optimal (maximum) enhancement. Moreover, large gaps are associated with significant influence of the sidewall surface to the capacitance (i.e. inset of Fig. 6); therefore, for any value of the gap, tall pillars are preferred for large enhancement. However, the maximal height and aspect ratio of the pillar is often limited by the fabrication process and the designer has much more flexibility in determining the diameter and spacing. Larger diameter pillars have larger sidewall surface areas, but lower sidewall surface area per unit substrate area, and therefore provide lower capacitance enhancement. This is also reflected in Eq. (10), where large diameter results in $1 /(1+S / D)^{2} \rightarrow 1$, but also decreases the term $g / D$. Therefore, in cases where the spacing and gap are sufficiently large (namely, satisfying the assumptions of the analytical model, Fig. 7), dense arrays are preferred.

An example of implementation of the analytical model is shown in Fig. 8, for the capacitance amplification of pillars with spacing of $S=100 \mu \mathrm{m}$, height of $H=150 \mu \mathrm{m}$ and gaps of $g=50 \mu \mathrm{m}$ (dashed lines) and $g=150 \mu \mathrm{m}$ (solid lines). Note that since relatively large spacing is used here, the analytical model is valid (Fig. 7). Our analysis shows that greater enhancement is obtained for taller pillars due to their large sidewall area. Moreover, maximal enhancement is achieved at $D / S$ ranging between 1 to 1.3 . For the case of no pillars, namely a flat surface with $H=0$, there is no amplification as expected. We note that though full optimization of the capacitance enhancement is beyond the scope of our work, based on our models and design and fabrication related limitations, such analysis can be carried out.

All geometries shown in Fig. 8 demonstrate capacitance enhancement for a gap of $g=150 \mu \mathrm{m}$. The enhancement ranges from tens to more than one hundred percent. The enhancement by micropillars is notably higher 
than that of perforated micro-plates, which only added several percent to the nominal value [10].

Continuing the design study, we find that the capacitance enhancement is significantly reduced for a smaller gap of $g=50 \mu \mathrm{m}$, and for some cases capacitance attenuation was achieved. This can be attributed to the significance of the contribution of the top surface in small gaps, as was discussed for the inset of Fig. 6, namely in this case the sidewalls and bottom contribution is significantly lower than that of the top. Capacitance attenuation was also found experimentally, see Array 4 in Fig. 6. Though Array 4 has a dense pillar configuration, namely high sidewall surface area per unit area or high ration $D / S$, the enhancement is rather low since the field lines from the pillars interact with one another, which reduces the contribution of the sidewalls to the total capacitance of the array.

Last, note that the capacitance of the topographies shown here is not to be compared directly to the performance of electrochemical or electrostatic supercapacitors that are sought in both miniaturized and macroscale systems $[13,14,15]$. As fixed electrode devices, supercapacitors are suited as energy storage rather than sensing or actuating devices that typically rely on moving electrodes. Nevertheless, the present study also shows how microstructures can increase capacitance by means of higher surface area, and the ability to achieve finer and higher aspect ratio surface features can benefit electrochemical and electrostatic energy storage as well.

\section{Conclusion}

In summary, we studied the enhancement to surface capacitance provided by an exemplary micro-scale topography of an array of cylindrical micropillars. Measurements on silicon substrates were validated by a full numerical solution of the ES-BVP as well as an approximate analytical model. We found that parametric variation of the array geometry can result in enhancement of the surface capacitance ranging from tens to more than one hundred percent, relative to a flat surface of the same area. However, certain pillar array dimensions result in attenuation of the capacitance, which emphasizes the importance of developing reliable modeling and design tools to obtain the desired performance. We conclude that micropillars and other topographies that are compatible with standard microfabrication processes could enable higher sensitivity capacitance measurements, both for metrology and MEMS device applications. Moreover, the ability to texture other materials such 


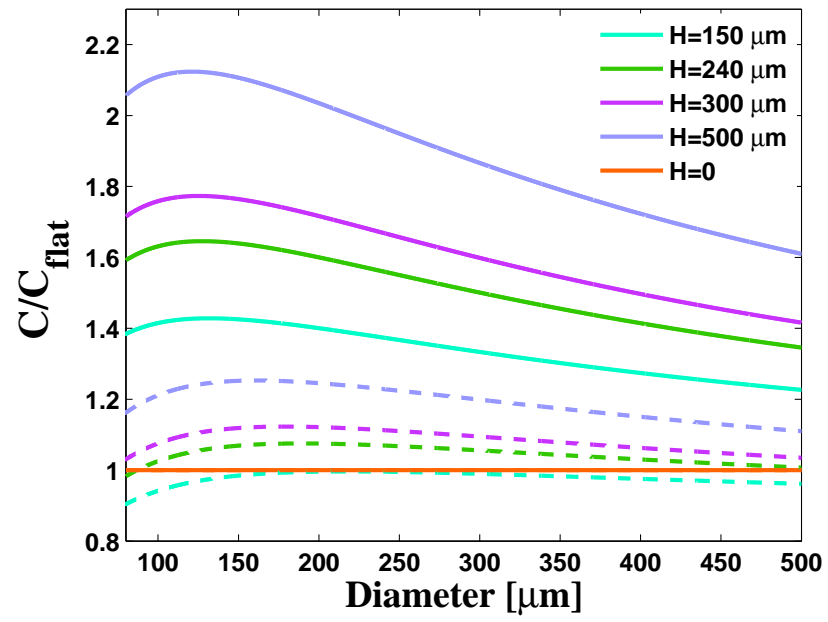

Figure 8: Capacitance enhancement versus the pillar diameter, for spacing of $S=100 \mu \mathrm{m}$ and different heights. Solid lines correspond to a gap of $g=150 \mu \mathrm{m}$ while dashed lines correspond to $g=50 \mu \mathrm{m}$.

as metal-coated and conducting polymers makes these findings relevant to tactile sensors for soft robotics and flexible electronics [4].

\section{Acknowledgments}

The authors thank Kendall Teichert, Justin Beroz, Mostafa Bedewy, Ryan Oliver and Tom Serbowicz for contributions to the design and building of the measurement setup. The authors also thank professor Isaac Harari from Tel Aviv University for inspiring discussion. Funding was provided by the Department of Energy Office of Basic Sciences (DE-SC0004927). Microfabrication was performed at the Lurie Nanofabrication Facility (LNF), which is a member of the National Nanotechnology Infrastructure Network (NNIN); and electron microscopy was performed at the Michigan Electron Microbeam Analysis Laboratory (EMAL).

\section{Reference}

[1] S. D. Senturia, Microsystem design, Vol. 3, Kluwer academic publishers Boston, MA, 2001. 
[2] C. Williams, W. Hough, S. Rishton, Scanning capacitance microscopy on a $25 \mathrm{~nm}$ scale, Applied physics letters 55 (2) (1989) 203-205.

[3] Y. Martin, D. Abraham, H. Wickramasinghe, High-resolution capacitance measurement and potentiometry by force microscopy, Applied Physics Letters 52 (13) (1988) 1103-1105.

[4] S. Mannsfeld, B. Tee, R. Stoltenberg, C. Chen, S. Barman, B. Muir, A. Sokolov, C. Reese, Z. Bao, Highly sensitive flexible pressure sensors with microstructured rubber dielectric layers, Nature materials 9 (10) (2010) 859-864.

[5] Z. Chen, R. Luo, Design and implementation of capacitive proximity sensor using microelectromechanical systems technology, Industrial Electronics, IEEE Transactions on 45 (6) (1998) 886-894.

[6] B. Yang, C. Lee, R. Kotlanka, J. Xie, S. Lim, A mems rotary comb mechanism for harvesting the kinetic energy of planar vibrations, Journal of Micromechanics and Microengineering 20 (6) (2010) 065017.

[7] H. Lee, S. Chang, E. Yoon, Dual-mode capacitive proximity sensor for robot application: Implementation of tactile and proximity sensing capability on a single polymer platform using shared electrodes, Sensors Journal, IEEE 9 (12) (2009) 1748-1755.

[8] R. C. Batra, M. Porfiri, D. Spinello, Electromechanical model of electrically actuated narrow microbeams, Journal of Microelectromechanical Systems 15 (5) (2006) 1175-1189. doi:10.1109/JMEMS.2006.880204.

[9] P. Bruschi, A. Nannini, F. Pieri, G. Raffa, B. Vigna, S. Zerbini, Electrostatic analysis of a comb-finger actuator with schwarz-christoffel conformal mapping, Sensors and Actuators A: Physical 113 (1) (2004) 106-117.

[10] A. Ya'akobovitz, S. Krylov, The influence of perforation on electrostatic and damping forces in thick SOI MEMS structures, Journal of Micromechanics and Microengineering 22 (11). doi:10.1088/0960$1317 / 22 / 11 / 115006$.

[11] L. Kogut, The influence of surface topography on the electromechanical characteristics of parallel-plate mems capacitors, Journal of Micromechanics and Microengineering 15 (5) (2005) 1068. 
[12] L. Jian, L. Ning, S. Yang, J. Wang, M. Hua, Triboelectrification Electrostatic Potential of MC Nylon 6 under Point Contact Dry Sliding, Tribology Letters 36 (3) (2009) 199-208. doi:10.1007/s11249-009-9473$\mathrm{z}$.

[13] L. L. Zhang, X. Zhao, Carbon-based materials as supercapacitor electrodes, Chemical Society Reviews 38 (9) (2009) 2520-2531.

[14] P. Banerjee, I. Perez, L. Henn-Lecordier, S. B. Lee, G. W. Rubloff, Nanotubular metal-insulator-metal capacitor arrays for energy storage, Nature Nanotechnology 4 (5) (2009) 292-296.

[15] A. Izadi-Najafabadi, S. Yasuda, K. Kobashi, T. Yamada, D. N. Futaba, H. Hatori, M. Yumura, S. Iijima, K. Hata, Extracting the full potential of single-walled carbon nanotubes as durable supercapacitor electrodes operable at $4 \mathrm{v}$ with high power and energy density, Advanced Materials 22 (35) (2010) E235-E241. 